Supplementary Information for

\title{
Variations in Atomic-Scale Step Edge Structures and Dynamics of Dissolving Calcite in Water Revealed by High-Speed Frequency Modulation Atomic Force Microscopy
}

\author{
Kazuki Miyata, ${ }^{a, b}$ Yuta Kawagoe, ${ }^{b}$ John Tracey ${ }^{c}$ Keisuke Miyazawa, ${ }^{b}$ \\ Adam S. Foster, ${ }^{a, b, c}$ Takeshi Fukuma ${ }^{*}, a, b$
}

\footnotetext{
${ }^{a}$ Nano Life Science Institute (WPI-NanoLSI), Kanazawa University, Kanazawa 920-1192, Japan

${ }^{b}$ Division of Electrical Engineering and Computer Science, Kanazawa University, Kanazawa 920-1192, Japan

${ }^{c}$ Department of Applied Physics, Aalto University, Helsinki FI-00076, Finland correspondence to: adam.foster@aalto.fi and fukuma@staff.kanazawa-u.ac.jp
}

This PDF file includes:

- Supplementary Figures

- Discussions on Possible Influence of Scan Artifacts 
(a)

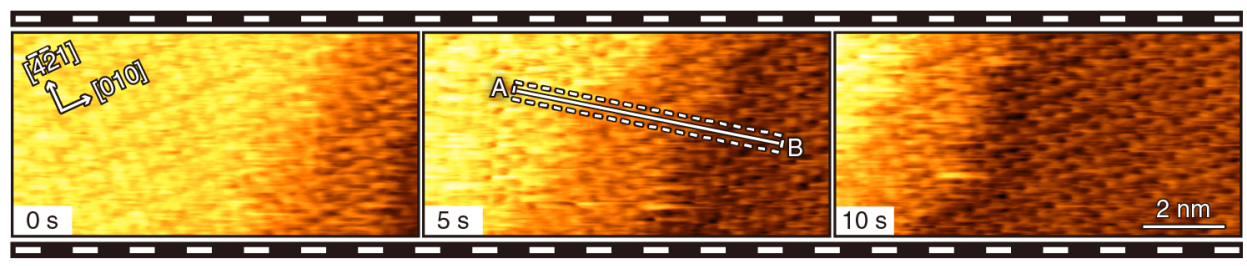

(b)

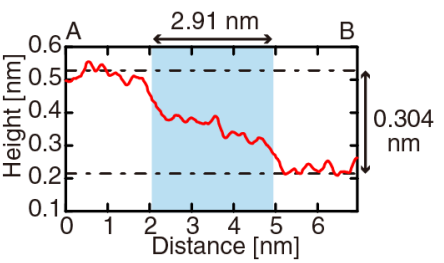

Figure S1. Backward (right to left) scan FM-AFM images of calcite (1014) surface in water. (a)

Backward scan images simultaneously obtained with the forward scan images shown in Fig. 1(b). (b) Height profiles measured along the line A-B.

(a)
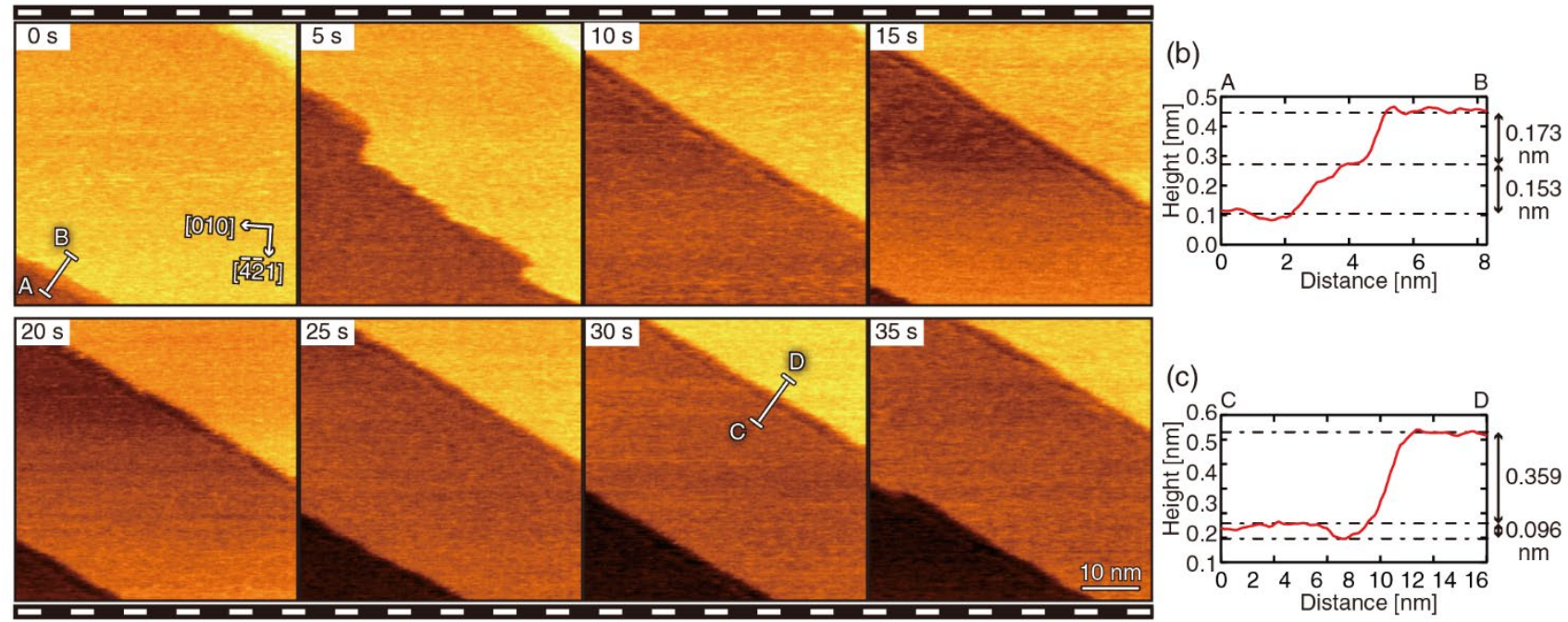

(d)

(e)
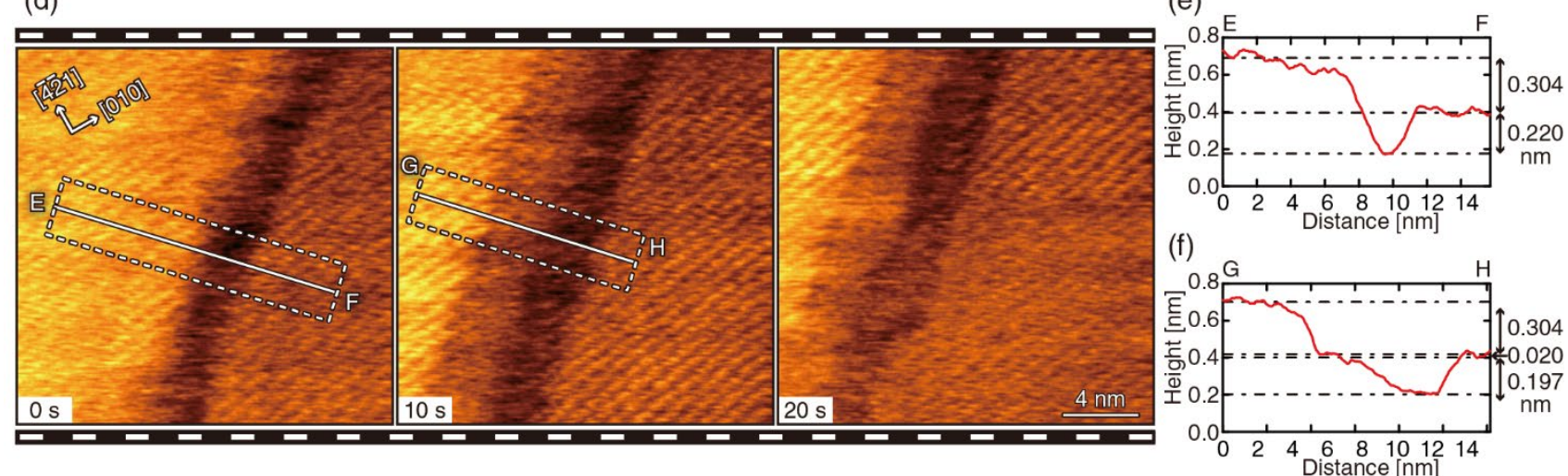

Figure S2. Backward (right to left) scan FM-AFM images of calcite (1014) surface in water. (a)

Backward scan images simultaneously obtained with the forward scan images shown in Fig. 3(a). (b,c)

Height profiles measured along the line A-B and C-D. (d) Backward scan images simultaneously

obtained with the forward scan images shown in Fig. 3(d). (e,f) Height profiles measured along the lines E-F and G-H. 
(a)

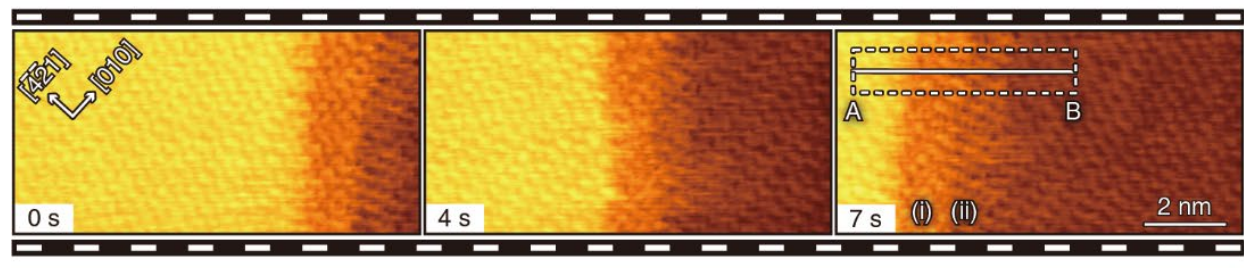

(c)

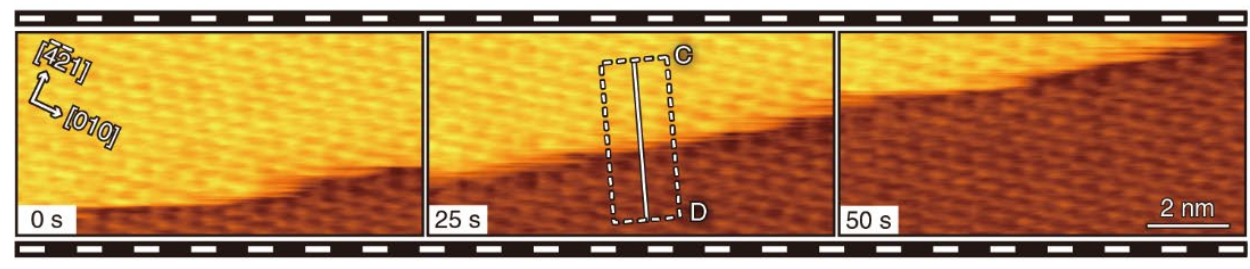

(b)

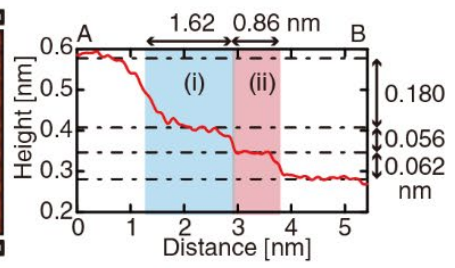

(d)

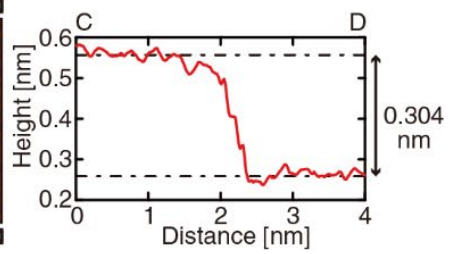

Figure S3. Backward (right to left) scan FM-AFM images of calcite (10ī4) surface in water. (a)

Backward scan images simultaneously obtained with the forward scan images shown in Fig. 4(a). (b)

Height profiles measured along the line A-B. (c) Backward scan images simultaneously obtained with the forward scan images shown in Fig. 3(d). (d) Height profiles measured along the line C-D. 


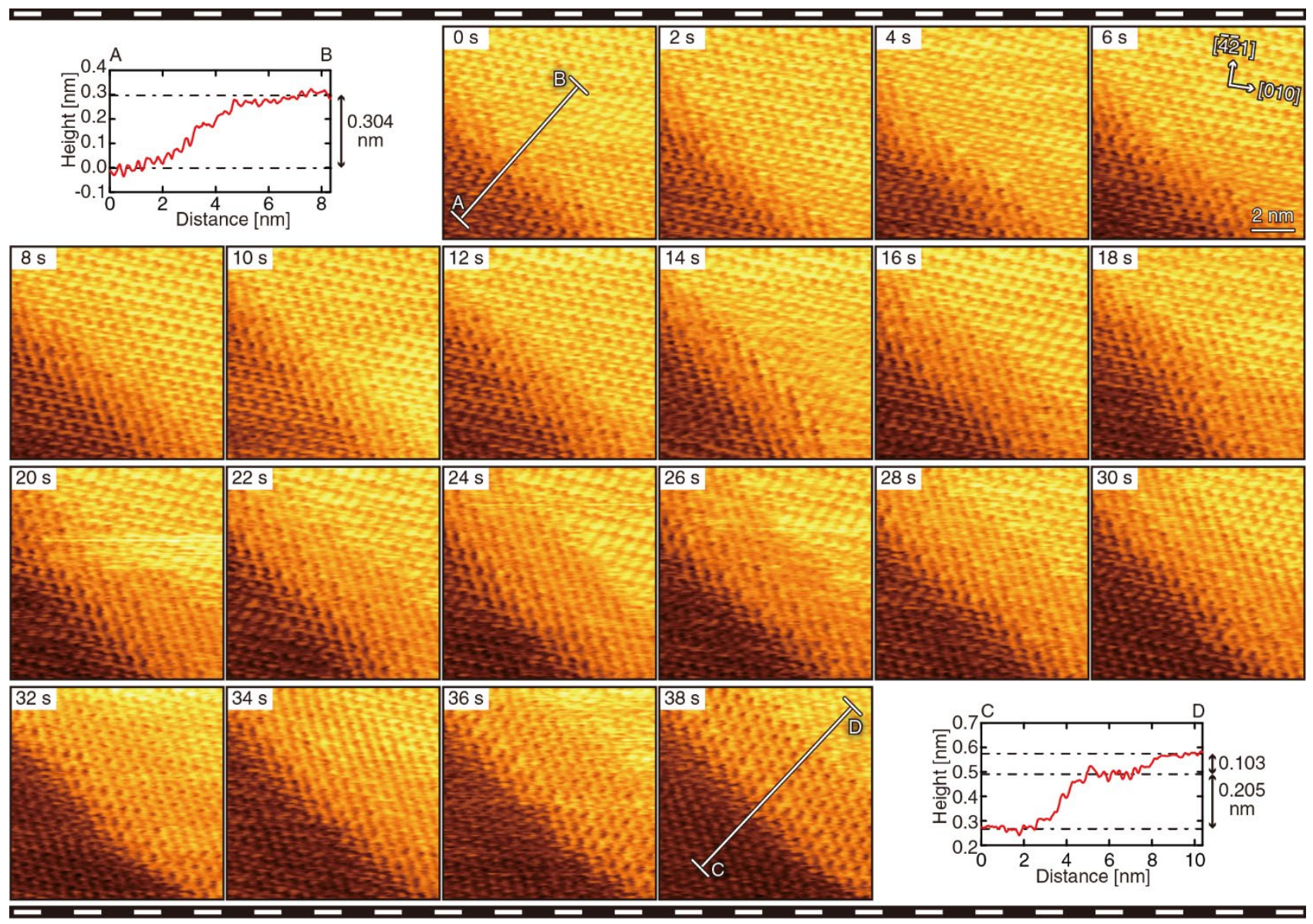

Figure S4. Backward (right to left) scan FM-AFM images of calcite (1014) surface in water obtained with the forward scan images shown in Fig. 5.

\section{(a) $\Delta f=1 \mathrm{kHz}$}

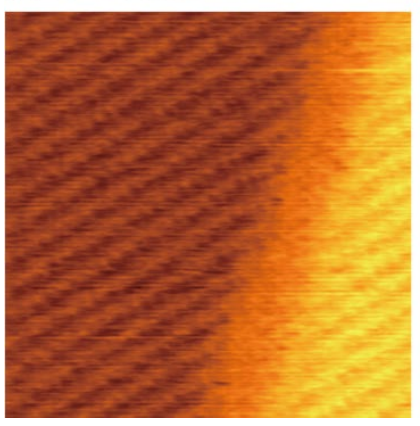

(b) $\Delta f=2 \mathrm{kHz}$

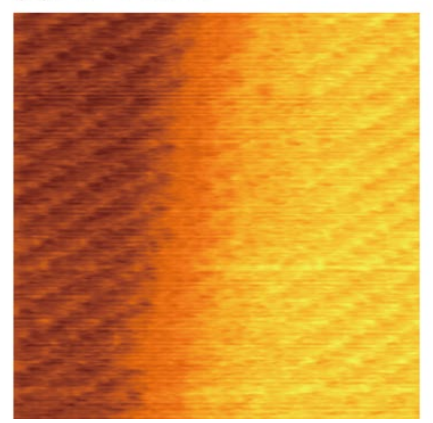

(c) $\Delta f=4 \mathrm{kHz}$

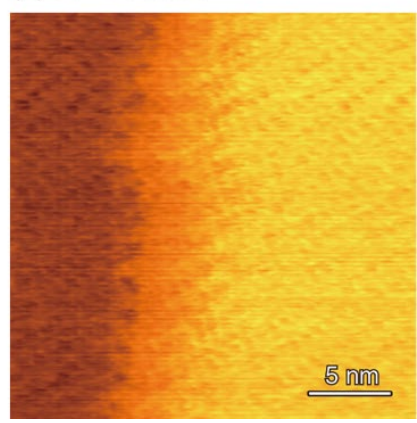

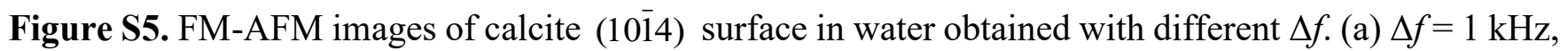
(b) $\Delta f=2 \mathrm{kHz},(\mathrm{c}) \Delta f=4 \mathrm{kHz}$. 


\section{Possible Influence of Scan Artifacts}

In the main text, we presented several variations of the step edge structures observed by FMAFM. It is widely known that atomic-scale contrasts observed in an AFM image can vary due to the artifacts caused by the feedback errors, set point drifts or non-ideal tip structures. Here, we explain why we consider that these possibilities are less likely than the proposed mechanisms.

The irregular structures at the step edges can be caused by overshooting or undershooting of the tip-sample distance feedback regulation if the feedback bandwidth is insufficient. However, we confirmed that the backward scan images (Figures S1-S4) show almost the same contrasts and height profiles as those observed in the forward scan images (Figures 1, 3, 4 and 5). Thus, this possibility can be safely excluded.

The FM-AFM images presented in this paper were obtained with the constant frequency shift mode where the tip-sample distance is regulated such that the frequency shift of the cantilever resonance $(\Delta f)$ is kept constant. On a heterogeneous surface, the $\Delta f$ versus distance curve shows site-dependent variations. Under such conditions, a slight change in the frequency shift set point can cause a significant change in the image contrast. In this study, we imaged the calcite step edge with different $\Delta f$ set points (Figure S5) within the range where we can obtain an atomic-scale image. The obtained images show little difference. Thus, it is unlikely that the drift of the $\Delta f$ set point can cause the observed variations in the step edge structures.

The atomic-scale AFM image contrasts often show strong dependence on the tip apex structure. In particular, at the step edges, if the tip has multiple protrusions close to each other, the steps can be imaged as multiple lines, which is called double tip effect. Thus, one may think that the observed transition regions (TRs) may be due to the double tip effect. However, as we explained in the previous publication [Nano Lett. 17 (2017) 4083], we consider such a possibility is very unlikely due to the reasons explained 
below. First, the observed TRs have a width of several nanometers, which is relatively wide for an atomicscale double tip effect. To explain it by the double tip effect, we need to assume multiple peaks on the tip apex with lateral and vertical separations of several nanometers and less than $0.2 \mathrm{~nm}$. It is clear that such a tip structure should be very rare. Of course, this can happen once in a while. However, as you can see from the images shown in this paper and statistical data shown in Fig. 6, we performed many experiments and have been able to reproducibly obtain similar images often. It is very unlikely that such a strange tip apex structure is formed so often. In addition, we presented direct imaging of the formation process of the TR in Fig. 5. These images show a gradual change in the step edge structure and did not show any discontinuous change in the contrast. This is very strong evidence to support that the TR was not due to the double tip effect, but represents the true surface structure.

Although we do not think the TRs are caused by the double tip effect, we do not exclude the possibility that some of the variation of the step edge structure may be caused by different tip apex structures. The $\Delta f$ versus distance curves are very sensitive to the tip apex structure as well as the surrounding solution conditions. Depending on the combination of these factors, the experimentally observed $\Delta f$ versus distance curves show wide variations. Even a minor change in the force profile can alter the selection of the tip trajectory from the many choices as explained in Fig. 2. These points are also discussed in the main text. 